\title{
Microfluidic device for robust generation of two-component liquid-in-air slugs with individually controlled composition
}

\author{
Kan Liu • Yi-Chun Chen · Hsian-Rong Tseng • \\ Clifton Kwang-Fu Shen • R. Michael van Dam
}

Received: 22 February 2010/Accepted: 5 April 2010/Published online: 22 April 2010

(C) The Author(s) 2010. This article is published with open access at Springerlink.com

\begin{abstract}
Using liquid slugs as microreactors and microvessels enable precise control over the conditions of their contents on short-time scales for a wide variety of applications. Particularly for screening applications, there is a need for control of slug parameters such as size and composition. We describe a new microfluidic approach for creating slugs in air, each comprising a size and composition that can be selected individually for each slug. Twocomponent slugs are formed by first metering the desired volume of each reagent, merging the two volumes into an end-to-end slug, and propelling the slug to induce mixing. Volume control is achieved by a novel mechanism: two closed chambers on the chip are initially filled with air, and a valve in each is briefly opened to admit one of the reagents. The pressure of each reagent can be individually selected and determines the amount of air compression, and thus the amount of liquid that is admitted into each chamber. We describe the theory of operation, characterize the slug generation chip, and demonstrate the creation of slugs of different compositions. The use of microvalves in
\end{abstract}

Kan Liu and Yi-Chun Chen contributed equally to this work.

Electronic supplementary material The online version of this article (doi:10.1007/s10404-010-0617-0) contains supplementary material, which is available to authorized users.

K. Liu · Y.-C. Chen · H.-R. Tseng · C. K.-F. Shen ( $\varangle)$ ·

R. M. van Dam $(\square)$

Department of Molecular \& Medical Pharmacology, Crump

Institute for Molecular Imaging, California NanoSystems

Institute, David Geffen School of Medicine, University

of California, Los Angeles, 570 Westwood Plaza, Los Angeles,

CA 90095, USA

e-mail: kshen@mednet.ucla.edu

R. M. van Dam

e-mail: mvandam@mednet.ucla.edu this approach enables robust operation with different liquids, and also enables one to work with extremely small samples, even down to a few slug volumes. The latter is important for applications involving precious reagents such as optimizing the reaction conditions for radiolabeling biological molecules as tracers for positron emission tomography.

Keywords Microfluidics · Liquid-in-air slug generation · Two-phase flow · PDMS · Programmable slug composition

\section{Introduction}

Microfluidic technology has made it possible to perform biochemical assays and chemical reactions in extremely tiny volumes. A particularly successful approach has been to use microdroplets or liquid slugs as individual microreactors, within which the reaction conditions can be very precisely controlled on short-time scales (Song et al. 2006). Applications that exploit the unique properties of droplets and slugs have been reported extensively in the literature, including optimization of tracer synthesis conditions for positron emission tomography (Olma et al. 2008), protein crystallization (Chao et al. 2004; Lau et al. 2007; Zheng et al. 2003), single-cell isolation and analysis (Sakai et al. 2004; Sugiura et al. 2007), micro- and nano-particle synthesis (Hung et al. 2006; Li et al. 2008, 2009a; Nie et al. 2005; Nisisako et al. 2006; Shestopalov et al. 2004; Yen et al. 2005), biochemical assays (Srisa-Art et al. 2007), and small-scale chemical reactions (Frenz et al. 2008; Ota et al. 2009; Park et al. 2009). Droplets and slugs have also been useful for increasing the interfacial area between the droplet/slug phase and an intervening carrier phase for performing multi-phase processes such as gas-liquid reactions 
(Doku et al. 2005; Gunther et al. 2005; Wada et al. 2006), liquid-liquid extractions (Jensen et al. 2001; Kralj et al. 2005, 2007), and distillations (Hartman et al. 2009), among others.

Every application depends critically on techniques and technologies that accurately generate the desired droplets or slugs, and there has been a flurry of research activity in this area (Teh et al. 2008). Many efforts have focused on continuous flow technologies for the generation of monodisperse droplets of fixed size and composition (Hsiung et al. 2006; Kim et al. 2006), with multi-component droplets formed either by injecting laminar streams into a carrier flow (Tice et al. 2004) or by forming each component as separate droplets that are later fused by controlling the downstream flow (Hung et al. 2006; Liu et al. 2007; Utada et al. 2005). In both the cases, mixing occurs rapidly during transport. Control of droplet size and composition has been achieved by dynamically controlling flow rates (Collins and Lee 2007) or by the integration of active modulation by, e.g., electric fields (Ristenpart et al. 2009), temperature (Nguyen et al. 2007), mechanical chopping (Hsiung et al. 2006), and piezoactuation (Pan et al. 2007) into chips. Droplets and slugs are most frequently generated in an oil carrier phase in such continuous flow devices, but liquid-in-gas and gas-inliquid slugs have also been demonstrated. Sophisticated screening assays have been performed by generating gradients of composition across many droplets (Laval et al. 2007; Zheng et al. 2003), or preparing sequences of precursor slugs in advance (Adamson et al. 2006; Hatakeyama et al. 2006; Li et al. 2006), but to the best of our knowledge, it remains a challenge to use these devices to efficiently (i.e., with low waste) generate droplets or slugs of independently selected composition when the total volume of reagents is small (e.g., tens to hundreds of slugs).

Another technology for creating droplets of controlled composition in air (or liquid) is digital microfluidics (Abdelgawad and Wheeler 2009; Chang and Yang 2007; Cho and Moon 2008), for example electrowetting-ondielectric (EWOD) devices. Feedback control techniques have been demonstrated that can accurately dispense liquid droplets of precise volume, and these single-component droplets can then be merged and mixed using wellestablished on-chip operations (Cho et al. 2003; Gong and Kim 2008). Microfluidic devices containing microvalves provide an alternative approach for the generation of slugs with chips that are easier to fabricate and control. Like in EWOD devices, timing of droplet formation can be accurately controlled, improving device reliability and facilitating automation. Generation of single-component slugs in air has been demonstrated (Bontoux et al. 2006; Gu et al. 2004; Thorsen et al. 2002), as have manipulations of slugs including merging and mixing (Hansen et al. 2004; Lau et al. 2007; Sassa et al. 2008; Zeng et al. 2009). The use of mechanically acting microvalves to physically separate slugs from the incoming reagent streams reduces the dependence of operation of liquid properties such as surface tension, viscosity, electrowetting response, etc.

Building on the microvalve approaches, we have developed a novel device for the generation of two-component liquid slugs that permits selection of composition over a continuous range that uses dead-end filling to avoid reagent waste, and that can work with slug volumes down to the 10-nl volume range. For each slug, reagents are metered by partially filling two chambers, the volume of each liquid determined by controlling the compression of gas that is initially trapped in each chamber. The liquid volumes are merged to form an end-to-end slug of the desired size and composition that can then be mixed by transporting it. This slug generator increases the degree of control over slug formation and can be combined with other on-chip functions to perform a variety of biological, chemical, and physical applications. An advantage of using integrated microvalves is that the incoming reagents can very easily be synchronized and slugs can be correctly generated starting with the very first one. This enables the use of slugs to perform screening of reaction conditions, even when the total volumes are very small (e.g., down to a few slug volumes). This device is ideally suited for screening applications with rare or expensive samples, such as enabling the optimization of reaction conditions of ${ }^{18} \mathrm{~F}$-labeled biomolecules as tracers for positron emission tomography (PET) to facilitate development and study of this rapidly emerging class of tracers (Liu et al. 2010).

\section{Experimental}

\subsection{Microfluidic chip}

The microfluidic slug generator (Fig. 1) was implemented as a two-layer polydimethylsiloxane (PDMS) chip according to common design and fabrication practices (Melin and Quake 2007). The upper layer of channels contains the fluidic channel network, consisting of reagent inlets, filling chambers, and slug output, while the lower layer contains microvalve control channels. Integrated "pinch valves" are created at each location where a control channel crosses directly below a fluid channel (Studer et al. 2004; Unger et al. 2000). The thin, elastic, PDMS membrane between the two channels can be actuated by applying pressure to the control channel, thus locally pinching off (closing) the fluid channel.

To fabricate the chip, photoresist-patterned silicon wafer molds for the fluid- and control-layers were first created by standard photolithographic techniques. Molds were pretreated by exposure to trimethylsilyl chloride (TMSCl) 

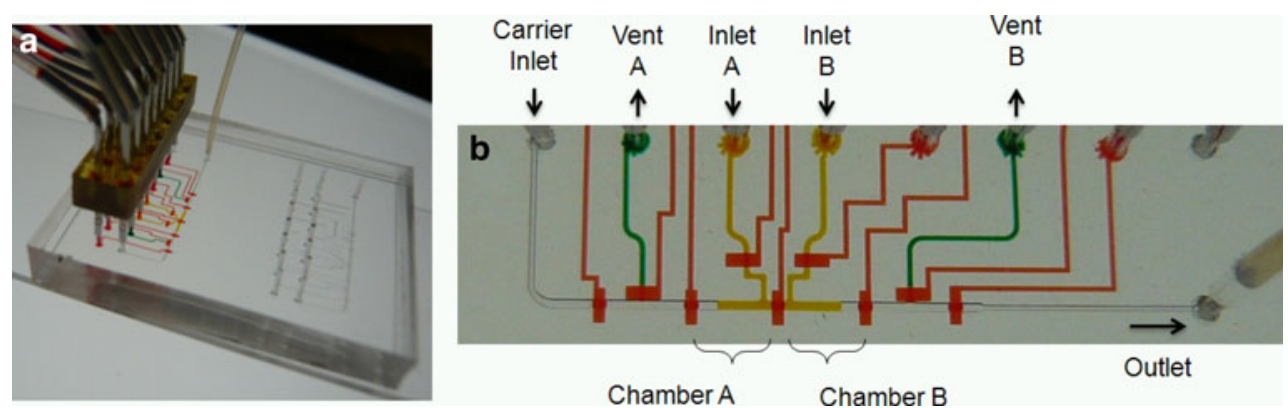

Fig. 1 a Photograph of PDMS microfluidic slug-generator. Tubing is connected to inlets and valve control channels via a custom connector developed in our laboratory. b Detail of slug generator. Control channels and microvalves have been filled with red food dye. Yellow food dye has been used to visualize the filling chambers $(A$ and $B)$ and associated reagent inlets. In this image, a slug of 1:1 ratio of liquids

vapor for $10 \mathrm{~min}$ to facilitate mold release. Well-mixed PDMS prepolymer (GE RTV615, component ratio A/B = $5: 1)$ was poured onto the fluid layer mold to give a $6-\mathrm{mm}$ thick fluidic layer with approximately $60-\mu \mathrm{m}$ channel depth. Another portion of PDMS prepolymer (GE RTV615, component ratio $\mathrm{A} / \mathrm{B}=20: 1$ ) was mixed and then spin-coated on the control layer mold at 1500 RPM for $60 \mathrm{~s}$. The fluidic and control layers were cured at $80^{\circ} \mathrm{C}$ for 15 and $18 \mathrm{~min}$, respectively, then aligned and then bonded by baking at $80^{\circ} \mathrm{C}$ for at least $6 \mathrm{~h}$. Holes were then punched into the assembly to form ports connected to liquid inlets and outlets in the fluidic layer, and to valve control channels in the control layer. Strong adhesion of the chip to a clean glass microscope slide to seal the control channels was achieved by corona discharge treatment (Enercon Industries Corp., Dyne-A-Mite HP) (Eddings et al. 2008). The microfluidic device was baked at $80^{\circ} \mathrm{C}$ for $72 \mathrm{~h}$ to restore the intrinsic hydrophobicity of PDMS surfaces required for manipulation of aqueous samples.

\subsection{Chip operation}

Fluids were delivered to the chip through PTFE microbore tubing, connected via short stainless steel tubes inserted directly into the chip. Liquids A and B were manually primed up to the valves V4 and V6 on the chip (Fig. 2) using the vent channels. Microvalve control lines were filled with water. On-chip valves were actuated by pressurizing the corresponding control channel to $410 \mathrm{kPag}$ via electronic solenoid valves (Series S070, SMC, Japan). The corresponding valve burst pressure was measured to be about $280 \mathrm{kPag}$, suggesting that fluid driving pressures up to about $210 \mathrm{kPag}$ could be reliably used in the chip. All valves were automatically controlled through a data acquisition module (USB-4750, Advantech, USA) driven by a custom software program written in LabView (National Instruments, USA).
A and B is being prepared. Each filling chamber is about $1400-\mu \mathrm{m}$ long, $300-\mu \mathrm{m}$ wide, and $60-\mu \mathrm{m}$ tall. Vent ports are shown in green. The slug generator is coupled to an outlet port in this chip, but would be directly coupled with other on-chip components for many applications

The sequence of valve states to operate the slug generator is shown in Fig. 2. The core of the chip consists of two adjacent chambers, $\mathrm{A}$ and $\mathrm{B}$, of fixed size (about 16-nl each) that can be isolated from the surrounding channels by microvalves, providing a means for precise volumetric metering of reagents. In the starting step, Step 1, all valves are sealed. Step 2 is used to ensure a well-defined air pressure, $P_{\text {initial }}$, initially inside each chamber. After the previous slug is ejected (as described below), the chambers contain air at pressure $P_{\text {initial }}=P_{\text {carrier }}$. Optionally, vent channels can be opened to release this pressure to atmosphere that establishes $P_{\text {initial }}=P_{\text {atm }}$. When used, venting was typically performed for $t_{\mathrm{vent}}=10 \mathrm{~s}$ to ensure sufficient time for pressures to equilibrate. Adjustment of $P_{\text {initial }}$ should be done immediately prior to Step 3 to prevent significant change in pressure due to gas permeation through the PDMS. In Step 3, two liquids, A and B, are filled into these chambers driven by pressures $P_{\text {fill, }}$ and $P_{\text {fill, }}$. The volume loaded into each chamber depends on $P_{\text {initial }}, P_{\text {fill }}$, and the filling time, $t_{\text {fill }}$. Because the chambers are isolated from one another during the filling process, and the three parameters can be independently adjusted for the two chambers, the volume of each liquid can be individually controlled, providing a means to dynamically tune the size and composition of every slug generated. After isolation of the two liquid volumes (Step 4), the chamber contents are merged together in Step 5 to form a single end-to-end slug. The process of sealing off the two chambers after filling isolates the measured liquids from the inlet streams, therefore decoupling this slug formation (merging) process from the properties and driving pressures of the two incoming liquids. In Step 6, the slug is pushed out of the chambers to the chip outlet with carrier gas (nitrogen) at pressure $P_{\text {carrier }}$. A slight narrowing of the downstream channel creates back-pressure that "cushions" the slug motion and prevents fragmentation of the slug that can occur when using relatively high $P_{\text {carrier }}$. Once the slug is ejected from the filling chambers, preparation of the next slug can begin. 

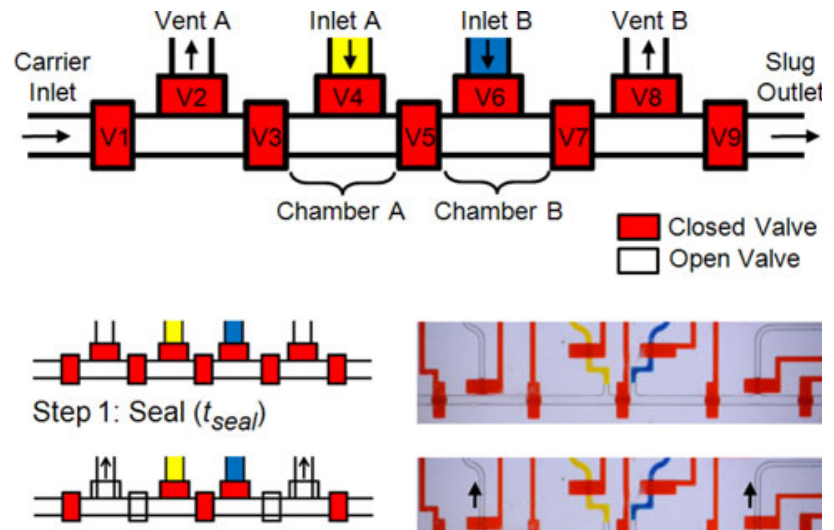

Step 2a (opt.): Vent ( $\left.t_{\text {vent }}\right)$

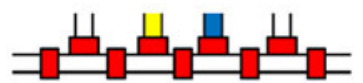

Step $2 \mathrm{~b}$ (opt.): Seal ( $\left.t_{\text {seal }}\right)$

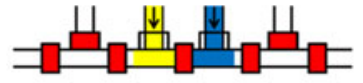

Step 3: Fill $\left(t_{\text {fill }}\right)$

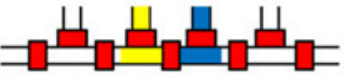

Step 4: Seal $\left(t_{\text {seal }}\right)$

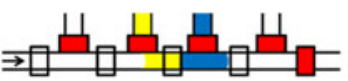

Step 5: Merge $\left(t_{\text {merge }}\right)$

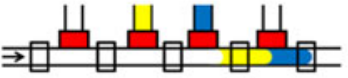

Step 6: Eject $\left(t_{\text {eject }}\right)$
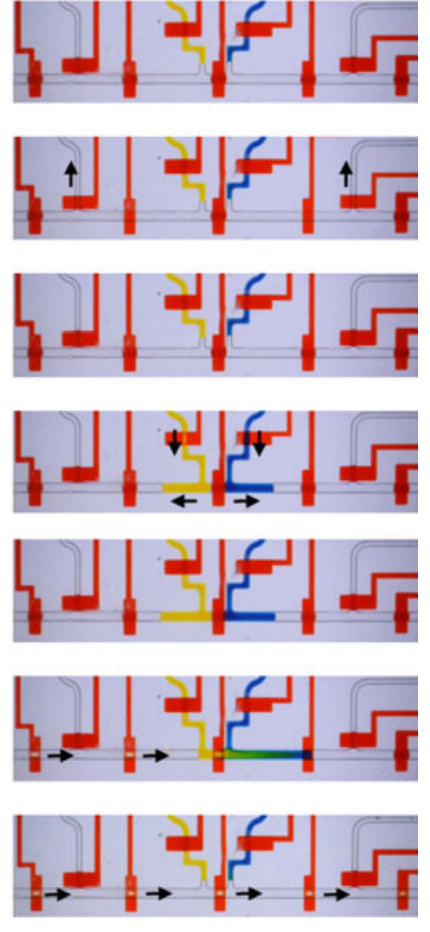

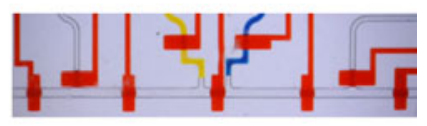

Fig. 2 (Top) schematic representation of slug generator. Two chambers $(A$ and $B)$ are separated by microvalve V5. Each chamber has a reagent inlet port and valve, and a vent outlet channel and valve in an adjacent chamber. Carrier (pressurized nitrogen) enters at the left, controlled by valve V1, to merge chamber contents and to push slugs to the outlet. (Bottom) detailed sequence of valve states for slug generation with duration shown in parentheses beside the name of the step. A micrograph of the actual chip generating slugs from colored liquids (water with food dye) is also shown for each step. The purpose of the sealing steps is to ensure that valves have sufficient time to close completely before other valves are opened $\left(t_{\text {seal }}=100 \mathrm{~ms}\right)$

Mixing of slugs occurs rapidly by an internal recirculation pattern as it is transported along the downstream fluid path (Rhee and Burns 2008). In this chip, slugs are injected sequentially into tubing. In many applications it may be preferable to couple the slug generator directly to other on-chip components such as reaction channels, (de)multiplexers, storage chambers, or sensors.

\subsection{Characterization}

The primary goal of this work is to develop an approach for reliable creation of two-component slugs with controlled composition. Since the composition is dictated by the amount of liquid filled into each chamber, we characterized the filling process as a function of adjustable parameters $\left(P_{\text {initial }}, P_{\text {fill }}\right.$, and $\left.t_{\text {fill }}\right)$ to determine the best approach for controlling slug composition.

Filling occurs independently in the two chambers due to the presence of microvalve V5, and therefore experiments could be performed using only a single chamber. In each experiment, the fraction of the chamber filled with liquid (filling fraction, $f$ ) was estimated by the ratio of the "length" of the liquid-filled portion to the total length of the chamber at any given time. Length observations were made by recording video of chip operation using a CCD camera (BigC Dino-Lite). The slug generator could be paused at will, permitting sufficient time to capture clear images without sophisticated high-speed visualization. Unless otherwise indicated, each data point is an average of three measurements from different slugs. Error bars represent the standard deviation. The largest source of error in these measurements was uncertainty in the length, due to factors such as an asymmetric meniscus. Observed filling fraction under various conditions was compared with theoretical predictions.

The PDMS channels bulge when filling pressure is applied (Holden et al. 2003), so to make accurate estimates of volume filled, the chamber contents were sealed after filling for $t_{\text {relax }}=5 \mathrm{~s}$, during which time the filled portion was observed to "lengthen" as trapped air escaped through the PDMS and the channel relaxed to its original crosssection. This ensures that all length measurements are made with the same channel cross-section and thus can be directly compared even if filling pressures are different.

\section{Results and discussion}

The microfluidic chip forms two-component slugs by merging the contents of two chambers containing reagents each with individually controlled volume. Partial dead-end filling of the chambers is studied in detail as a means to enable dynamic control of slug size and composition.

\subsection{Time dependence of filling}

First, the temporal aspects of the filling process were studied. Using a single chamber, filling fraction of deionized water was measured as a function of filling time, $t_{\text {fill }}$ (Fig. 3). Filling was observed to proceed in two distinct phases with very different time scales. Initially, within tens of milliseconds, the chamber fills to a certain fraction (shown later to depend on filling pressure, $P_{\text {fill }}$ ). The second phase is much slower (tens of seconds) and appears approximately linear. The combination of very high and very low slopes makes it difficult to use $t_{\text {fill }}$ as a means to 
a

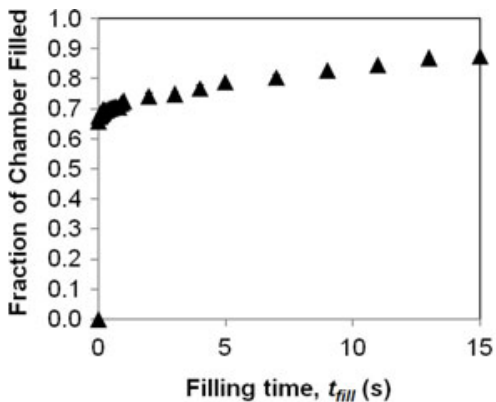

b

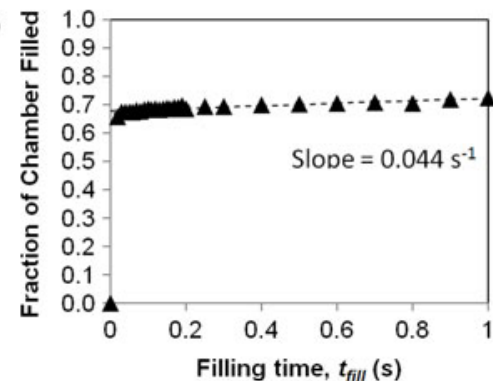

Fig. 3 a Fraction filled of one slug generator chamber with deionized water as a function of filling time $\left(t_{\text {fill }}\right)$ at $P_{\text {fill }}=140 \mathrm{kPag}$, illustrating the time evolution of the filling process. $P_{\text {carrier }}$ was $60 \mathrm{kPag}$ and $P_{\text {initial }}$ was $0 \mathrm{kPag}$ (i.e., using venting) for all measurements. b Detail of filling for the first $1 \mathrm{~s}$. c Conceptual model of two-phase filling process. (i) Initially the chamber is filled with air. (ii) During the first

control the fraction filled over a wide range. However, the insensitivity to time (e.g., $4.4 \%$ per second for the first $1 \mathrm{~s}$ in Fig. 3) does have an advantage: if $t_{\text {fill }}$ is chosen within the second phase, filling fraction will not be sensitive to small inaccuracies in filling time (e.g., tens to hundreds of milliseconds) that may arise due to factors such as electronics delays and finite valve response time.

We chose to operate our device with a fixed filling time, selected just past the transition between the two phases, i.e., $t_{\text {fill }}=100 \mathrm{~ms}$. Keeping the filling time short permits higher slug generation rates, and also ensures the filling fraction is close to its value at the end of the fast phase, which can be predicted with good accuracy (see below). The 100-ms filling time was chosen based on data collected with deionized water. For other liquids, it may be necessary to choose a slightly longer filling time to ensure the slow filling phase is reached. An example of filling with glycerol is described in the Supplementary Information.

\subsection{Ideal gas model of filling process}

Filling appears to occur by rapid compression of the trapped gas initially in the chamber (fast phase), followed by permeation of this pressurized gas out through the PDMS matrix (slow phase). In the fast compression phase, the final steady-state "plateau" that is reached can be predicted from the ideal gas law, independent of any fluid

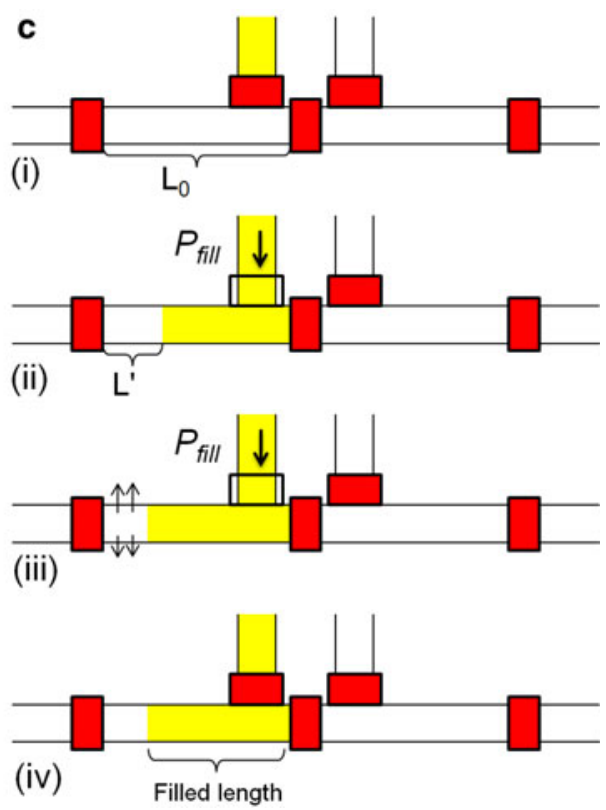

filling phase, the air is rapidly compressed, allowing part of the chamber to be filled with liquid. (iii) In the second phase, the compressed air slowly leaks through the PDMS chip, permitting additional liquid to enter the chamber. (iv) Finally, the inlet valve is closed, trapping the liquid in the chamber

properties or device geometry. We ignore the dynamics of how quickly the steady state is achieved, which would involve factors that affect fluid movement, such as viscosity, geometry of channel and interconnect, filling pressure, and total liquid volume. (This issue is addressed in the Supplementary Information where it is shown that by choosing a slightly longer $t_{\text {fill }}$ these dynamics can be ignored.)

The fraction of the chamber filled at the end of the fast compression phase can be modeled as a function of $P_{\text {fill }}$ and $P_{\text {initial }}$. Assume that a chamber of volume $V_{0}$ is initially filled with air at pressure $P_{\text {initial }}$. When filling begins, this gas rapidly compresses to a steady-state pressure $P_{\text {fill }}$. Assuming there is negligible loss of gas or temperature change, the ideal gas law should hold, and the final volume of compressed air is:

$V^{\prime}=V_{0} \frac{P_{\text {initial }}}{P_{\text {fill }}}$

The remaining volume of the chamber is now filled with liquid from the inlet channel. Thus for the given pressures, the fraction filled with liquid at the end of the compression phase is expected to be:

$f=\frac{V_{0}-V^{\prime}}{V_{0}}=1-\frac{P_{\text {initial }}}{P_{\text {fill }}}$

This simple model has no fitted parameters. 


\subsection{Pressure dependence of filling}

Equation 2 predicts that with a fixed filling time selected just after the end of the compression phase, the filling fraction depends on both the filling pressure $P_{\text {fill }}$ and the initial pressure, $P_{\text {initial }}$. Experimentally, the expected dependence on both the pressures is seen (see Fig. 4a, b), and comparison with the ideal gas model (solid line) after conversion of gauge to absolute pressures of Eq. 2 shows good agreement at low $P_{\text {fill }}$. However, there is increasing discrepancy between experimental data and the model at higher filling pressures, presumably due to the expansion of the PDMS channel under pressure. Modeling of this effect is discussed in the next section.

Both curves are monotonic and have moderate slope; thus, either of these two pressures, $P_{\text {fill }}$ or $P_{\text {initial }}$, can be manipulated to control the filling fraction of liquids, and thus the composition of slugs. Linear dependence on $P_{\text {initial }}$ makes $P_{\text {initial }}$ preferable in principle, but because it is difficult to accurately maintain pressure inside a PDMS chip due to the high permeability of the PDMS material, we chose to use $P_{\text {fill }}$ as a control variable instead. $P_{\text {initial }}$ was fixed at 0 $\mathrm{kPag}$ by venting the chambers prior to filling to create the next slug. Negative pressure was considered and would provide a greater range of filling fractions, but it cannot be as accurately maintained as atmospheric pressure $(0 \mathrm{kPag})$.

Two different filling pressures, $P_{\text {fill,A }}$ and $P_{\text {fill, B }}$, for the two incoming liquids can be used to tune dynamically the degree of filling of each, thereby determining the composition and size of the liquid slugs generated. Using a fast electronic pressure regulator to change driving pressures, it is feasible to select the size and the composition of every slug individually. Over the filling pressure ranges used, we achieved fractions filled covering the range from about 0.1 to 0.8 as shown in Fig. 4, enabling slugs composed of $\mathrm{A} / \mathrm{B}=1: 8$ to $8: 1$ to be created.

\subsection{Effect of capillary force}

The model of Eq. 2 assumes that the initially trapped air is compressed to a pressure equal to the filling pressure, $P_{\text {fill }}$. However, if the liquid-air interface is not flat during filling,
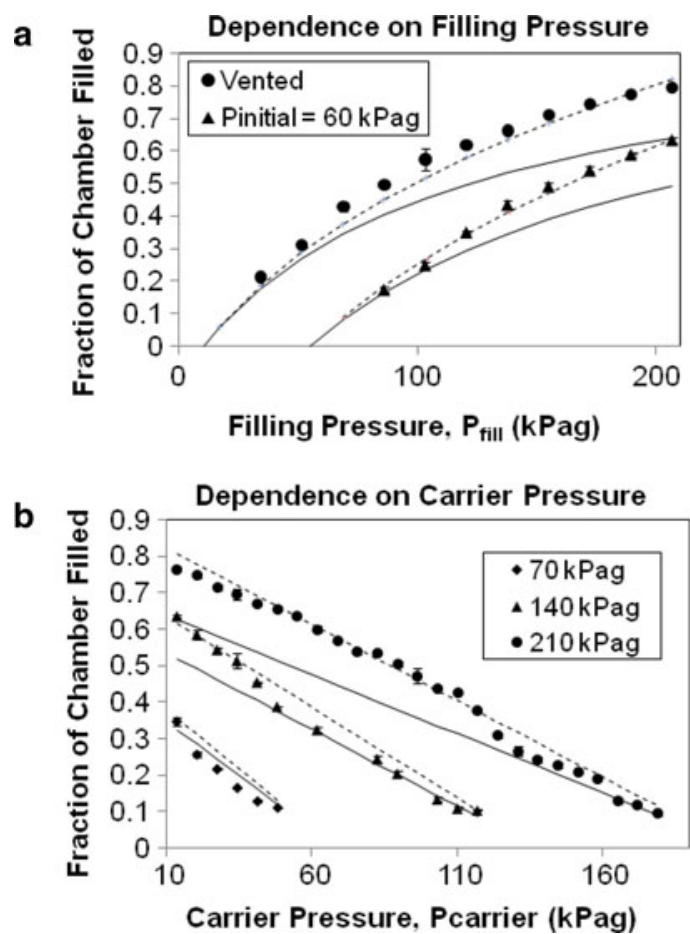

Fig. 4 a Fraction filled of one slug generator chamber with water as a function of filling pressure, $P_{\text {fill }}$, at fixed $t_{\text {fill }}=100 \mathrm{~ms}$ and fixed $P_{\text {initial }}=60 \mathrm{kPag}\left(P_{\text {carrier }}\right)$ or $0 \mathrm{kPag}$ (vented). Solid lines show the theoretical ideal gas model for the cases of $P_{\text {initial }}=60$ and $10 \mathrm{kPag}$. The latter resulted in closer agreement than with $P_{\text {initial }}=0 \mathrm{kPag}$, suggesting that in the experiment the pressure was not completely vented prior to filling (only 5-s venting time was used). Dotted lines represent the model with modifications for PDMS expansion. b Fraction filled of one slug generator chamber with water as a function of initial pressure, $P_{\text {initial }}$, at fixed $P_{\text {fill }}$ of 70,140 and 210 $\mathrm{kPag}$, and fixed $t_{\text {fill }}=100 \mathrm{~ms}$. Solid lines represent the ideal gas

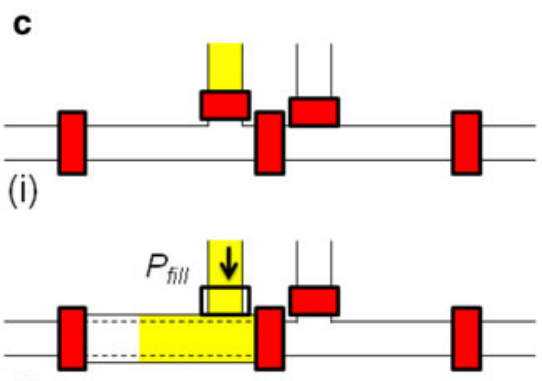

(ii)

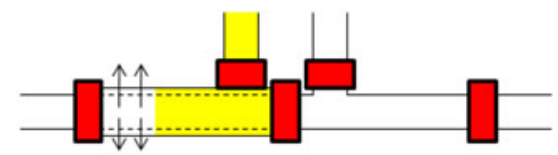

(iii)

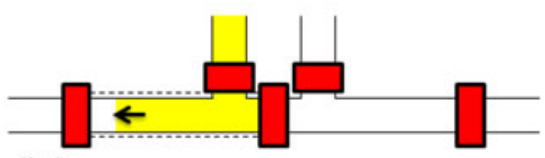

(iv)

model for the selected fixed filling pressures, and the dotted lines represent the theory with modifications for PDMS expansion. c Conceptual model of PDMS channel expansion and the effect on filling fraction. (i) Initially chamber is filled with air. (ii) The chamber is filled to a fraction, $f$, but the channel cross-section is increased (due to elastic deformation by internal pressure $P_{\text {fill }}$ ) so the absolute amount of liquid in the chamber is larger would be expected if there is no expansion. (iii) Air remaining in the chamber after filling escapes via permeation reducing the pressure. (iv) The channel relaxes to its original cross-section, distributing the liquid to fill a longer portion of the channel. It is the relaxed length that is measured 
an additional capillary pressure is generated (Geerken et al. 2007; Li et al. 2009b). The result of this correction would be a lateral shift in plots such as Fig. 4a. We estimate the magnitude, based on the properties of water and PDMS as well as the device geometry, to be on the order of $1 \mathrm{kPa}$ or less. The capillary pressure would therefore have only very small effect on filling fraction (for the range of filling pressures used in this study), and therefore is ignored.

\subsection{Correcting for PDMS expansion}

The low elastic modulus of PDMS results in substantial deformation of channel dimensions at high internal pressure (Gervais et al. 2006; Holden et al. 2003). The channel cross-section expands during filling due to the application of pressure $P_{\text {fill }}$. The volume fraction filled still follows the ideal gas model, but because the cross-section is larger, the chamber actually contains more liquid than if the channel did not expand. The magnitude of the discrepancy depends on the change in cross-sectional size which increases with on filling pressure. This is evident in the data in Fig. $4 \mathrm{a}$ and b that exceed the simple ideal gas model that neglects the expansion.

To ensure that all data points were comparable, so that length of the filled portion reflects the volume fraction of the original-sized chamber, we measured filled lengths after permitting chambers to relax to original dimensions. During this delay, trapped air permeates out of the chamber through the PDMS, gradually reducing the pressure and thus the deformation (see Fig. 4c). As the channel dimensions decrease, the liquid is redistributed to fill a longer length of the channel.

To model the discrepancy between actually amount filled and the prediction of Eq. 2, we estimate the equilibrium deformation of the chamber under the filling pressure, $P_{\text {fill }}$, and make a correction to the expected length fraction that accounts for the extra volume filled due to this deformation. The geometry of this problem can be roughly approximated as a thick-walled elastic cylinder that is internally pressurized, where the chamber/channel radius represents the inner radius of the cylinder, the bulk PDMS of the chip represents the wall of the cylinder, and the outside of the chip represents the outer radius of the cylinder. Though chips in reality are not cylinders centered around microchannels we in fact find that the functional form of this model has good agreement with the experimental data.

The model predicts (Wedgwood 1928) that the channel experiences a radial strain that is linearly related to the internal pressure $P$ :

$\varepsilon_{r}=k P$ where $k$ is an expression that depends on the elastic modulus and Poisson's ratio of the PDMS as well as geometry of the "cylinder."

The cross-sectional area of the channel therefore enlarges to $A^{\prime \prime}=A_{0}\left(1+\varepsilon_{r}\right)^{2}=A_{0}\left(1+k P_{\text {fill }}\right)^{2}$, where $A_{O}$ is the original cross-section. The ideal gas model predicts that a fraction $f$ of this larger chamber will be filled. We can express the total volume filled as $f A^{\prime \prime} L_{0}=f\left(1+k P_{\text {fill }}\right)^{2}$ $A_{0} L_{0}=f^{\prime \prime} A_{0} L_{0}$. After relaxation to the original channel size $A_{0}$, the measured length fraction can therefore be predicted as:

$f^{\prime \prime}=f\left(1+k P_{\text {fill }}\right)^{2}=\left(1-\frac{P_{\text {initial }}}{P_{\text {fill }}}\right)\left(1+k P_{\text {fill }}\right)^{2}$

where $f$ is the prediction of the ideal gas model from Eq. 2 .

This model, plotted in Figs. 4 and 5 (dotted lines), has one fitted parameter, $k$, and shows good agreement with the data when $k=0.00065 \mathrm{kPag}^{-1}$. The dependence on, and sensitivity to, the material and geometrical properties should be investigated in more detail to ensure operational parameters remain constant from chip to chip. As a preliminary test, we performed experiments with several different chips of varying ages and observed only about $1 \%$ discrepancy in chamber filling fractions (data not shown).

\subsection{Generation of two-component slugs}

We have shown above how to control the filling fraction of a single chamber by varying $P_{\text {fill }}$. Under the conditions used in this article, the fraction filled depends only on the ideal gas law, and an adjustment for the expansion of PDMS channels under pressure that depends on PDMS properties and geometry. There should be no dependence on fluid properties such as viscosity, contact angles, total volume, etc., other than brief transient effects during the initial part of the filling process.

For a desired slug size and ratio of components, it is possible to determine (from data in Fig. 4 or Eq. 4) what filling pressures should be used. We applied our microfluidic chip to the creation of slugs with arbitrarily selected compositions in the range 8:1-1:8. Figure 5 illustrates an example where several different slug compositions were generated by maintaining a constant filling pressure of liquid A (water), $P_{\text {fill, }}=140 \mathrm{kPag}$, while varying the filling pressure of liquid B (water), $P_{\text {fill,B }}$ from 20 to 170 $\mathrm{kPag}$. Compositions access in this experiment ranged from about $\mathrm{A} / \mathrm{B}=4.5-0.9$.

Using food dye in water, end-to-end slugs were qualitatively observed to be well mixed within a few slug lengths of movement through the channel downstream of the slug generator. We did not quantitatively analyze the 


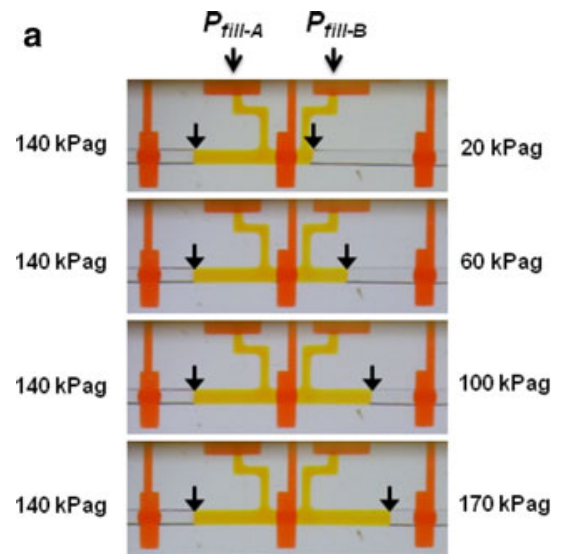

Fig. 5 a Photographs of slug generator after completion of the filling step for different combinations of filling pressures $P_{\text {fill, }}$ (liquid A in left chamber) and $P_{\text {fill,B }}$ (liquid B in right chamber). b Generation of two-component slugs with different compositions. Inlet A was kept at a constant pressure, $P_{\text {fill, }}=140 \mathrm{kPag}$, and inlet B was varied over a range of pressures, $P_{\text {fill, B }}$. $P_{\text {carrier }}$ was $60 \mathrm{kPag}, P_{\text {initial }}$ was $0 \mathrm{kPag}$

mixing because this principle has been studied by numerous researchers (Rhee and Burns 2008).

An important application of the ability to controllably generate tiny mixtures in this fashion is in optimizing reaction conditions with precious reagents. As an example, ongoing research efforts in our laboratory are investigating the use of the slug generator for radiolabeling of biological molecules for use as molecular imaging tracers in positron emission tomography (PET). In these reactions, the labeling yield is very sensitive to parameters such as concentrations, $\mathrm{pH}$, and type/sequence of biomolecule (Liu et al. 2010). To maximize the yield, both to conserve biomolecule and to achieve superior quality images, one generally must perform trial-and-error optimization. At the conventional scale $(100 \mathrm{~s}$ of $\mu \mathrm{l})$ this would consume enormous amounts of biomolecule and so optimization is rarely done, resulting often in poor yield. However, in this microfluidic device reaction mixtures could be prepared on demand in truly tiny volumes ( $10 \mathrm{~s}$ of $\mathrm{nl}$ ), each of which can be reacted and analyzed by existing off-chip analytical tools to guide the optimization process. The space of reaction parameters could then be explored quite extensively with only minimal reagent consumption.

\subsection{Comparison to other slug-generation methods}

Unlike continuous flow devices, where the process of droplet and slug formation is affected by properties and flow rates of the incoming streams as well as the contents of the downstream channel (thus depending on the history of operation), formation of slugs in our device is insulated from these effects. Isolating the chambers from the liquid inlets after filling decouples slug formation (merging) from

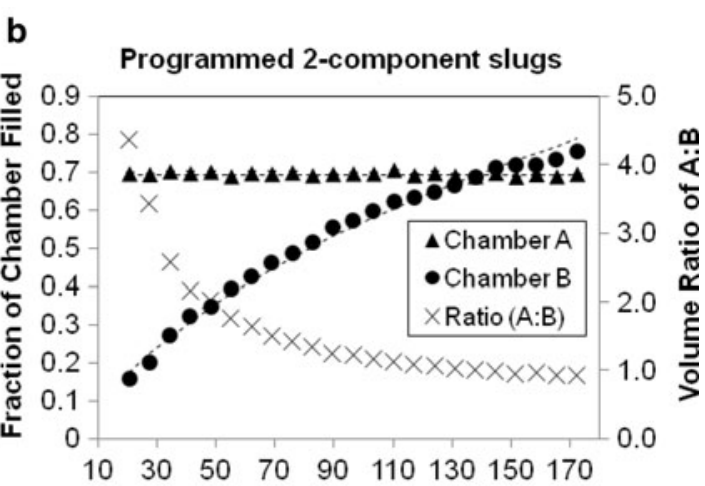

Chamber B Filling Pressure, $\boldsymbol{P}_{\text {fill-B }}(\mathrm{kPag})$

(vented), and $t_{\text {fill }}$ was $100 \mathrm{~ms}$. The fraction of chambers A and B that are filled in each case are shown, along with a dotted line representing the theoretical ideal gas model with modification for PDMS expansion. The volume ratio of the two components in each resulting slug, $\mathrm{A} / \mathrm{B}$, is shown on the secondary $y$-axis

the properties and driving pressures of the two incoming liquids. In addition, isolation of the chambers from the downstream channel decouples the filling and slug formation process from the history of operation. These properties are what enable instant selection of new slug composition at any time without a transition period that wastes reagents. They also permit slug generation to start and stop on demand, making it easier to synchronize slug formation and integrate with other subsystems in an automated fashion.

Despite these advantages, the approach presented here may not be suitable for certain applications, due to limitations in operating speed and fluid compatibility. Operating speed is limited by the microvalve response time and is significantly slower than the high slug generation rates that can be achieved in some continuous approaches; however, it is still quite acceptable for a wide range of screening applications, especially those involving relatively small numbers of slugs or where the slug analysis time is long. Fluid compatibility is limited by the use of PDMS which swells in many organic solvents (Lee et al. 2003). It may be possible to expand the range of applications by fabricating similar devices with inert materials such as perfluoropolyether (Rolland et al. 2004) or hybrids of glass and Teflon (Grover et al. 2008).

\subsection{Further developments}

Improvements in accuracy of volume ratios might be achieved using non-permeable, non-elastic materials for the filling chamber. This would improve the ability to accurately maintain $P_{\text {initial, }}$, and would also eliminate the channel expansion that currently introduces some dependence of 
slug generation on the mechanical properties of the chip material.

Our lab is currently investigating a different approach for improving ratio accuracy that retains the advantages of fabricating chips from PDMS. This approach will be based on the concept of quantized filling fractions, achieved by the use of valves to subdivide each chamber into several subchambers that are each either completely filled or empty. We are also exploring the extension of these concepts to accurate generation of slugs composed of three or more reagents.

\section{Conclusion}

We have demonstrated and characterized a microfluidic device for the robust generation of multi-component liquidin-air slugs for applications such as screening of reaction conditions involving precious reagents. The device is a proof of concept demonstrating that microfluidic platforms with integrated microvalves can produce slug flows with the advantage of programmable size and composition of slugs. Selected volumes of two reagents are loaded into separate chambers and then merged together into a single end-to-end slug that can be efficiently mixed by transport along the downstream microchannel. The slug is separated from the input reagent streams by mechanically actuating valves, decoupling the slug formation process from the influence of fluid parameters. Slugs are also isolated from the downstream channel during formation. Two-component slug generation using our approach should therefore be more predictable and robust than other methods of slug formation over a range of reagents. A simple model of the volume-control process was reported and found to be in excellent agreement with experimental data. A key advantage of the chip reported here is that it can operate reliably even with very small total volumes of the two reagents $(<1 \mu \mathrm{l})$ and with minimal waste of reagents. Accurate slug formation begins with the very first slug and can be continued until the very last one. Size and composition can be programmed individually for each slug, with no transition period during which slugs are malformed, such as the stabilization time needed in continuous flow approaches. Straightforward extensions to our design could enable the formation of programmable slugs comprising three or more reagents.

Acknowledgments This study was supported in part by the National Cancer Institute (Nanosystems Biology Cancer Center, Grant number U54CA119347-01), the Industry-University Cooperative Research Program (UC Discovery Grant number bio07-10665) and a project development grant from the UCLA Center for In Vivo Imaging in Cancer Biology (National Institutes of Health Grant number 5P50CA086306). The authors thank Dirk Williams and Darin Williams for assistance in designing and fabricating custom chip connectors.

Open Access This article is distributed under the terms of the Creative Commons Attribution Noncommercial License which permits any noncommercial use, distribution, and reproduction in any medium, provided the original author(s) and source are credited.

\section{References}

Abdelgawad M, Wheeler AR (2009) The digital revolution: a new paradigm for microfluidics. Adv Mater 21(8):920-925

Adamson DN, Mustafi D, Zhang JXJ, Zheng B, Ismagilov RF (2006) Production of arrays of chemically distinct nanolitre plugs via repeated splitting in microfluidic devices. Lab Chip 6(9):11781186

Bontoux N, Pepin A, Chen Y, Ajdari A, Stone HA (2006) Experimental characterization of hydrodynamic dispersion in shallow microchannels. Lab Chip 6(7):930-935

Chang CC, Yang RJ (2007) Electrokinetic mixing in microfluidic systems. Microfluid Nanofluid 3(5):501-525

Chao W-C, Collins J, Wang S-W, Li GP, Bachman M, Lee AP (2004) Control of concentration and volume gradients in microfluidic droplet arrays for protein crystallization screening. In: Proceedings of the 26th annual international conference of the IEEE EMBS. San Francisco, CA, USA, pp 2623-2626

Cho SK, Moon H (2008) Electrowetting on dielectric (EWOD): new tool for bio/micro fluids handling. Biochip J 2(2):79-96

Cho SK, Moon HJ, Kim CJ (2003) Creating, transporting, cutting, and merging liquid droplets by electrowetting-based actuation for digital microfluidic circuits. J Microelectromech Syst 12(1):70 80

Collins J, Lee AP (2007) Control of serial microfluidic droplet size gradient by step-wise ramping of flow rates. Microfluid Nanofluid 3(1): 19-25

Doku GN, Verboom W, Reinhoudt DN, van den Berg A (2005) Onmicrochip multiphase chemistry-a review of microreactor design principles and reagent contacting modes. Tetrahedron 61(11):2733-2742

Eddings MA, Johnson MA, Gale BK (2008) Determining the optimal PDMS-PDMS bonding technique for microfluidic devices. J Micromech Microeng 18(6):1-4

Frenz L, El Harrak A, Pauly M, Begin-Colin S, Griffiths AD, Baret JC (2008) Droplet-based microreactors for the synthesis of magnetic iron oxide nanoparticles. Angew Chem Int Ed 47(36):6817-6820

Geerken MJ, Lammertink RGH, Wessling M (2007) Interfacial aspects of water drop formation at micro-engineered orifices. J Colloid Interface Sci 312(2):460-469

Gervais T, El-Ali J, Gunther A, Jensen KF (2006) Flow-induced deformation of shallow microfluidic channels. Lab Chip 6(4):500-507

Gong J, Kim CJ (2008) All-electronic droplet generation on-chip with real-time feedback control for EWOD digital microfluidics. Lab Chip 8(6):898-906

Grover WH, von Muhlen MG, Manalis SR (2008) Teflon films for chemically-inert microfluidic valves and pumps. Lab Chip 8(6):913-918

Gu W, Zhu XY, Futai N, Cho BS, Takayama S (2004) Computerized microfluidic cell culture using elastomeric channels and Braille displays. Proc Natl Acad Sci USA 101(45):15861-15866 
Gunther A, Jhunjhunwala M, Thalmann M, Schmidt MA, Jensen KF (2005) Micromixing of miscible liquids in segmented gas-liquid flow. Langmuir 21(4):1547-1555

Hansen CL, Sommer MOA, Quake SR (2004) Systematic investigation of protein phase behavior with a microfluidic formulator. Proc Natl Acad Sci USA 101(40):14431-14436

Hartman RL, Sahoo HR, Yen BC, Jensen KF (2009) Distillation in microchemical systems using capillary forces and segmented flow. Lab Chip 9(13):1843-1849

Hatakeyama T, Chen DLL, Ismagilov RF (2006) Microgram-scale testing of reaction conditions in solution using nanoliter plugs in microfluidics with detection by MALDI-MS. J Am Chem Soc 128(8):2518-2519

Holden MA, Kumar S, Beskok A, Cremer PS (2003) Microfluidic diffusion diluter: bulging of PDMS microchannels under pressure-driven flow. J Micromech Microeng 13(3):412-418

Hsiung SK, Chen CT, Lee GB (2006) Micro-droplet formation utilizing microfluidic flow focusing and controllable moving-wall chopping techniques. J Micromech Microeng 16(11):2403-2410

Hung LH, Choi KM, Tseng WY, Tan YC, Shea KJ, Lee AP (2006) Alternating droplet generation and controlled dynamic droplet fusion in microfluidic device for CdS nanoparticle synthesis. Lab Chip 6(2):174-178

Jensen H, Fermin DJ, Girault HH (2001) Photoinduced electron transfer at liquid/liquid interfaces. Part V. Organisation of watersoluble chlorophyll at the water/1,2-dichloroethane interface. Phys Chem Chem Phys 3(12):2503-2508

Kim SJ, Song YA, Skipper PL, Han J (2006) Electrohydrodynamic generation and delivery of monodisperse picoliter droplets using a poly(dimethylsiloxane) microchip. Anal Chem 78(23):80118019

Kralj JG, Schmidt MA, Jensen KF (2005) Surfactant-enhanced liquidliquid extraction in microfluidic channels with inline electricfield enhanced coalescence. Lab Chip 5(5):531-535

Kralj JG, Sahoo HR, Jensen KF (2007) Integrated continuous microfluidic liquid-liquid extraction. Lab Chip 7(2):256-263

Lau BTC, Baitz CA, Dong XP, Hansen CL (2007) A complete microfluidic screening platform for rational protein crystallization. J Am Chem Soc 129(3):454-455

Laval P, Lisai N, Salmon JB, Joanicot M (2007) A microfluidic device based on droplet storage for screening solubility diagrams. Lab Chip 7(7):829-834

Lee JN, Park C, Whitesides GM (2003) Solvent compatibility of poly(dimethylsiloxane)-based microfluidic devices. Anal Chem 75(23):6544-6554

Li L, Mustafi D, Fu Q, Tereshko V, Chen DLL, Tice JD, Ismagilov RF (2006) Nanoliter microfluidic hybrid method for simultaneous screening and optimization validated with crystallization of membrane proteins. Proc Natl Acad Sci USA 103(51):1924319248

Li SW, Xu HH, Wang YJ, Luo GS (2008) Controllable preparation of nanoparticles by drops and plugs flow in a microchannel device. Langmuir 24(8):4194-4199

Li SW, Xu JH, Wang YJ, Luo GS (2009a) Liquid-liquid two-phase flow in pore array microstructured devices for scaling-up of nanoparticle preparation. Aiche J 55(12):3041-3051

Li SW, Xu JH, Wang YJ, Luo GS (2009b) A new interfacial tension measurement method through a pore array micro-structured device. J Colloid Interface Sci 331(1):127-131

Liu K, Ding HJ, Chen Y, Zhao XZ (2007) Droplet-based synthetic method using microflow focusing and droplet fusion. Microfluid Nanofluid 3(2):239-243

Liu K, Lepin EJ, Wang M-W, Guo F, Lin W-Y, Chen Y-C, Sirk SJ, Olma S, Phelps ME, Zhao X-Z, Tseng H-R, van Dam RM, Wu AM, Shen CK-F (2010) Microfluidic-based 18F-labeling of biomolecules for ImmunoPET (under review)
Melin J, Quake SR (2007) Microfluidic large-scale integration: the evolution of design rules for biological automation. Annu Rev Biophys Biomed 36:213-231

Nguyen NT, Ting TH, Yap YF, Wong TN, Chai JCK, Ong WL, Zhou J, Tan SH, Yobas L (2007) Thermally mediated droplet formation in microchannels. Appl Phys Lett 91(8):084102

Nie ZH, Xu SQ, Seo M, Lewis PC, Kumacheva E (2005) Polymer particles with various shapes and morphologies produced in continuous microfluidic reactors. J Am Chem Soc 127(22):80588063

Nisisako T, Torii T, Takahashi T, Takizawa Y (2006) Synthesis of monodisperse bicolored janus particles with electrical anisotropy using a microfluidic co-flow system. Adv Mater 18(9):1152-1156

Olma S, Liu K, Chen Y-C, Tseng HR, van Dam RM, Shen CKF (2008) Application of a microfluidic droplet mixer for [18F]fluorine labeling of biomolecules for positron emission tomography. In: Proceedings of the twelfth international conference on miniaturized systems for chemistry and life sciences, pp 604606

Ota S, Yoshizawa S, Takeuchi S (2009) Microfluidic formation of monodisperse, cell-sized, and unilamellar vesicles. Angew Chem Int Edit 48(35):6533-6537

Pan CT, Shiea J, Shen SC (2007) Fabrication of an integrated piezoelectric micro-nebulizer for biochemical sample analysis. J Micromech Microeng 17(3):659-669

Park JI, Nie Z, Kumachev A, Abdelrahman AI, Binks BR, Stone HA, Kumacheva E (2009) A microfluidic approach to chemically driven assembly of colloidal particles at gas-liquid interfaces. Angew Chem Int Edit 48(29):5300-5304

Rhee M, Burns MA (2008) Drop mixing in a microchannel for lab-ona-chip platforms. Langmuir 24(2):590-601

Ristenpart WD, Bird JC, Belmonte A, Dollar F, Stone HA (2009) Non-coalescence of oppositely charged drops. Nature 461(7262):377-380

Rolland JP, Van Dam RM, Schorzman DA, Quake SR, DeSimone JM (2004) Solvent-resistant photocurable "liquid teflon" for microfluidic device fabrication. J Am Chem Soc 126(8):2322-2323

Sakai S, Kawabata K, Ono T, Ijima H, Kawakami K (2004) Preparation of mammalian cell-enclosing subsieve-sized capsules $(<100 \mathrm{mu} \mathrm{m})$ in a coflowing stream. Biotechnol Bioeng 86(2):168-173

Sassa F, Fukuda J, Suzuki H (2008) Microprocessing of liquid plugs for bio/chemical analyses. Anal Chem 80(16):6206-6213

Shestopalov I, Tice JD, Ismagilov RF (2004) Multi-step synthesis of nanoparticles performed on millisecond time scale in a microfluidic droplet-based system. Lab Chip 4(4):316-321

Song H, Chen DL, Ismagilov RF (2006) Reactions in droplets in microfluidic channels. Angew Chem Int Ed 45(44):7336-7356

Srisa-Art M, deMello AJ, Edel JB (2007) High-throughput DNA droplet assays using picoliter reactor volumes. Anal Chem 79(17):6682-6689

Studer V, Hang G, Pandolfi A, Ortiz M, Anderson WF, Quake SR (2004) Scaling properties of a low-actuation pressure microfluidic valve. J Appl Phys 95(1):393-398

Sugiura S, Oda T, Aoyagi Y, Matsuo R, Enomoto T, Matsumoto K, Nakamura T, Satake M, Ochiai A, Ohkohchi N et al (2007) Microfabricated airflow nozzle for microencapsulation of living cells into 150 micrometer microcapsules. Biomed Microdevices 9(1):91-99

Teh SY, Lin R, Hung LH, Lee AP (2008) Droplet microfluidics. Lab Chip 8(2): 198-220

Thorsen T, Maerkl SJ, Quake SR (2002) Microfluidic large-scale integration. Science 298(5593):580-584

Tice JD, Lyon AD, Ismagilov RF (2004) Effects of viscosity on droplet formation and mixing in microfluidic channels. Anal Chim Acta 507(1):73-77 
Unger MA, Chou HP, Thorsen T, Scherer A, Quake SR (2000) Monolithic microfabricated valves and pumps by multilayer soft lithography. Science 288(5463):113-116

Utada AS, Lorenceau E, Link DR, Kaplan PD, Stone HA, Weitz DA (2005) Monodisperse double emulsions generated from a microcapillary device. Science 308(5721):537-541

Wada Y, Schmidt MA, Jensen KF (2006) Flow distribution and ozonolysis in gas-liquid multichannel microreactors. Ind Eng Chem Res 45(24):8036-8042

Wedgwood GA (1928) The elastic properties of thick cylindrical shells under internal pressure. Proc Phys Soc 41:366-383
Yen BKH, Gunther A, Schmidt MA, Jensen KF, Bawendi MG (2005) A microfabricated gas-liquid segmented flow reactor for hightemperature synthesis: the case of CdSe quantum dots. Angew Chem Int Ed 44(34):5447-5451

Zeng SJ, Li BW, Su XO, Qin JH, Lin BC (2009) Microvalve-actuated precise control of individual droplets in microfluidic devices. Lab Chip 9(10):1340-1343

Zheng B, Roach LS, Ismagilov RF (2003) Screening of protein crystallization conditions on a microfluidic chip using nanolitersize droplets. J Am Chem Soc 125(37):11170-11171 